This item was submitted to Loughborough's Research Repository by the author.

Items in Figshare are protected by copyright, with all rights reserved, unless otherwise indicated.

\title{
Nonlinear amplification in a second-harmonic waveguide free-electron laser
}

PLEASE CITE THE PUBLISHED VERSION

PUBLISHER

(C) IEEE

VERSION

VoR (Version of Record)

LICENCE

CC BY-NC-ND 4.0

\section{REPOSITORY RECORD}

Zhong, Xiehe, and Michael G. Kong. 2019. "Nonlinear Amplification in a Second-harmonic Waveguide Freeelectron Laser". figshare. https://hdl.handle.net/2134/5221. 
This item was submitted to Loughborough's Institutional Repository (https://dspace.lboro.ac.uk/) by the author and is made available under the following Creative Commons Licence conditions.

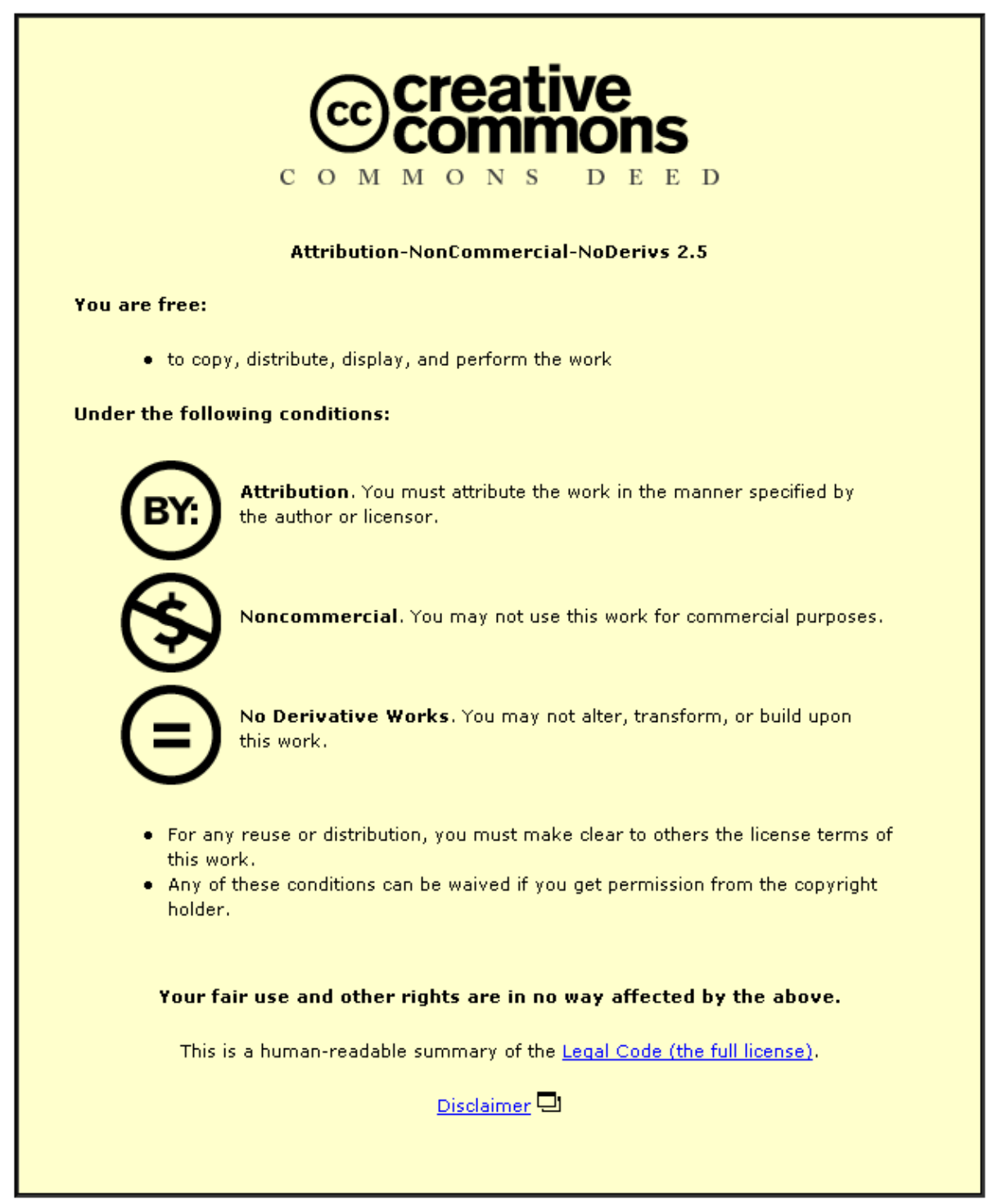

For the full text of this licence, please go to: http://creativecommons.org/licenses/by-nc-nd/2.5/ 


\title{
Nonlinear Amplification in a Second-Harmonic Waveguide Free-Electron Laser
}

\author{
Xiehe Zhong, Student, IEEE, and Michael G. Kong, Senior Member, IEEE
}

\begin{abstract}
This paper describes the results of numerical simulation of second-harmonic waveguide free-electron lasers (FELs) from the small-signal regime to the large-signal regime. Aimed at reducing the size and hence the cost of compact waveguide FELs operated from the microwave to the far infrared, these unconventional waveguide FELs can substantially decrease the minimum electron energy required for strong FEL radiation at a given frequency while increasing the small-signal gain. This contribution focuses on their saturation behaviors, taking into consideration variation in wiggler field and electron-energy spread. Depending on the roundtrip power loss within the FEL cavity and the initial electron-energy spread, the computed relationship between interaction gain and in-cavity power can be used to maximize the output power at a given electron current. Furthermore, it is found that gain degradation due to electron-energy spread remains relatively unchanged regardless of radiation power and wiggler field.
\end{abstract}

Index Terms-Free electron lasers, harmonic generation, masers.

\section{INTRODUCTION}

$\mathbf{T}$ HERE is increasing interest to develop low-cost and compact free-electron lasers (FELs) from the microwave to far infrared regions [1]-[6]. These are typically driven by low-current electron beams and are useful for numerous applications that do not require coherent radiation power above tens of kilowatts. To minimize the size and hence the cost of these low-current FELs, it is important to reduce the minimum electron energy required for strong FEL radiation at a given frequency [2]. In addition to the obvious advantage of using smaller and less expensive power supplies, FEL operation at reduced electron energy also increases the interaction gain indirectly through its inverse proportion to $\gamma^{5} \beta^{5}$ [2].

As a technique to reduce electron-energy, second-harmonic waveguide FELs based on a novel longitudinal interaction mechanism have been considered recently [7]-[9]. In such unconventional beam-wave interaction configuration, electrons acquire first in the wiggler field a rapid longitudinal velocity oscillation twice as fast as their transverse velocity oscillation. Hence their subsequent interaction with the $E_{z}$ component of TM modes in a cylindrical waveguide may be considered as in an effective wiggler having a period half as much as that of the actual wiggler. It was shown analytically that the electron energy required for radiation at a given frequency can be reduced significantly in second-harmonic waveguide FELs from that needed in comparable conventional waveguide

Manuscript received March 15, 2002; revised July 8, 2002.

The authors are with the Department of Electronic and Electrical Engineering, Loughborough University, Leicestershire LE11 3TU, U.K.

Digital Object Identifier 10.1109/TPS.2002.807507
FELs, where the transverse electron velocity couples with the transverse electric-field component of TE modes in a rectangular waveguide [7], [8]. Second-harmonic waveguide FELs are also different from conventional waveguide FELs in that they have a nonresonant electron velocity component that couples with the resonant velocity component to contribute significantly to the interaction gain [8]. This unique feature may be exploited to enhance the beam-wave interaction and to control the radiation spectrum. Furthermore, for the preferred operation mode of large wiggler field, the beam-wave interaction in second-harmonic waveguide FELs is studied in detail and formulated to the eighth order of the wiggler field using both Madey's theorem and perturbation theory [9].

These small-signal studies lead to the question whether the radiation field with large small-signal gain continues to grow strongly and yield a sizeable output power. To this end, secondharmonic waveguide FELs need to be studied in the large-signal regime where laser amplification becomes nonlinear and eventually, the radiation field ceases to grow any further. Effects of the wiggler field and initial electron energy also need to be studied in detail. In Section II, a numerical model describing saturation behavior of second-harmonic waveguide FELs is discussed. Based on this model, nonlinear interaction in secondharmonic waveguide FELs is studied with different wiggler field strengths. In Section III, we consider the maximum attainable output power from the FEL cavity as a function of wiggler field and a function of cavity loss. Then in Section IV, we will consider effects of initial energy spread in the large signal regime before summarize our conclusions in Section V.

\section{Numerical Model for Large-Signal Simulation}

Second-harmonic waveguide FELs employ an unconventional configuration of paring a planar wiggler magnet with a cylindrical waveguide as shown in Fig. 1. In this study we will analyze such FEL systems with a one-dimensional (1-D) treatment. In the 1-D limit, the $\mathrm{TM}_{01}$ waveguide mode has only one on-axis field component and this is the axial electric field given below

$$
\vec{E}_{z}=\hat{z} E_{0} \cos \left(k_{z} z-\omega t+\varphi\right) .
$$

In practice, $\mathrm{TE}$ modes (particularly the $\mathrm{TE}_{01}$ mode) will compete with the $\mathrm{TM}_{01}$ mode in their excitation in the cylindrical waveguide and so need to be suppressed. One technique to enhance excitation for $\mathrm{TM}_{01}$ mode while suppressing low $\mathrm{TE}$ modes is to add thin and straight axial slots on the inner wall of the waveguide. These will disrupt the surface current induced 


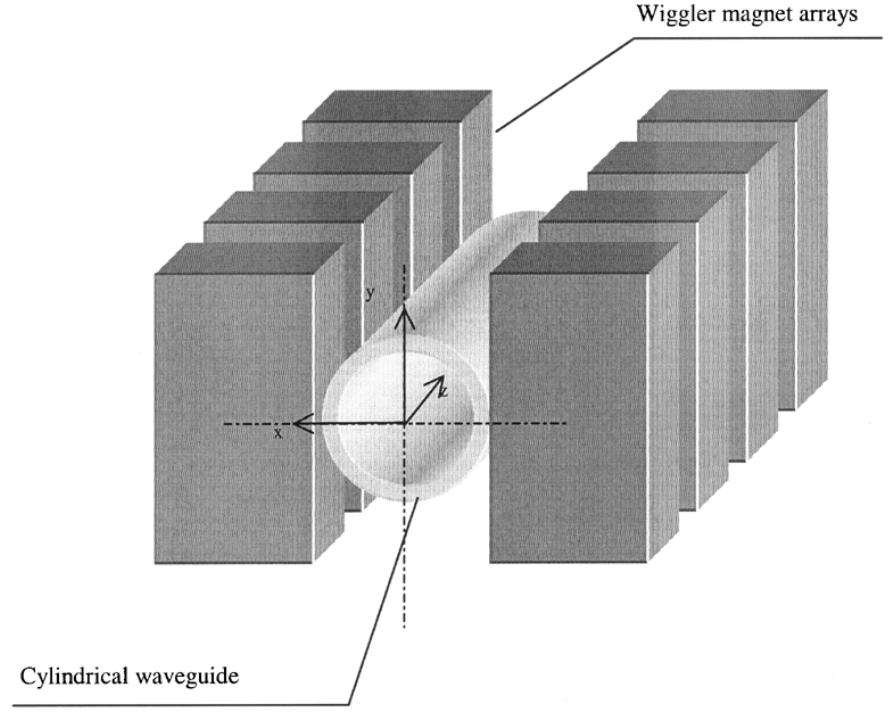

Fig. 1. Schematic illustration of the second-harmonic waveguide FEL configuration.

by the electric-field component of TE modes at the waveguide wall and thus discourage their excitation. Similarly, the wiggler field is also approximated by its on-axis expression of

$$
\vec{B}_{w}=\hat{x} B_{w 0} \cos \left(k_{w} z\right) .
$$

in the 1-D limit. For the beam-wave interaction, we assume that the magnitude of the radiation field remains unchanged for each passage of the electron beam through the FEL interaction region. By repeating computation of such single-pass interaction at different radiation fields (or input power levels), the dynamic evolution of the electron induced radiation field in an FEL cavity can be modeled and unravelled. From the small-signal analysis of second-harmonic waveguide FELs, it has been shown that the axial electron velocity can be Taylor expanded to the eighth order of the wiggler field that [8], [9]

$$
\beta_{z}=\beta_{z 0}\left(1+\chi_{0} \cos 2 k_{w} z\right)
$$

where

$$
\begin{aligned}
\beta_{z 0} & =\hat{\beta}_{0}\left[1-\frac{1}{16}\left(\frac{a_{w r}^{2}}{\gamma_{0}^{2} \hat{\beta}_{0}^{2}}\right)^{2}-\frac{15}{1024}\left(\frac{a_{w r}^{2}}{\gamma_{0}^{2} \hat{\beta}_{0}^{2}}\right)^{4}\right] \\
\chi_{0} & =\frac{\hat{\beta}_{0}}{\beta_{z 0}}\left[\frac{1}{2} \frac{a_{w r}^{2}}{\gamma_{0}^{2} \hat{\beta}_{0}^{2}}+\frac{3}{64}\left(\frac{a_{w r}^{2}}{\gamma_{0}^{2} \hat{\beta}_{0}^{2}}\right)^{3}\right] \\
\hat{\beta}_{0} & =\sqrt{1-\left(1+a_{w r}^{2}\right) / \gamma_{0}^{2}}
\end{aligned}
$$

where $a_{w r}=e B_{w 0} / m_{0} k_{w} \sqrt{2}$ is the root mean square (RMS) value of the normalized wiggler field. Similar to other FEL devices, the electron dynamics in second-harmonic waveguide FELs are governed by the energy conservation equation and the relativistic Newton-Lorentz equations

$$
\begin{aligned}
\frac{d\left(\gamma m c^{2}\right)}{d t} & =-e(\vec{E} \cdot \vec{v}) \\
\frac{d(\gamma m \vec{v})}{d t} & =-e(\vec{E}+\vec{v} \times \vec{B})
\end{aligned}
$$

where $\gamma$ is the relativistic Lorentz factor, $\vec{v}$ is the electron velocity, $\vec{E}$ is the electric field component of the electron producing radiation, and $\vec{B}$ is the combined magnetic field of both the wiggler magnet and the propagating radiation wave. As beam-wave interactions in compact waveguide FELs are generally weak, it is usually adequate to approximate both $\vec{E}$ and $\vec{B}$ with their on-axis components [4], [7]-[9]. Therefore, one can obtain the following equations for second-harmonic waveguide FELs in the 1-D limit:

$$
\begin{aligned}
& \frac{d\left(\gamma m c^{2}\right)}{d t}=-e\left(\vec{E}_{z} \cdot \vec{v}_{z}\right) \\
& \frac{d\left(\gamma m \vec{v}_{z}\right)}{d t}=-e\left(\vec{E}_{z}+\vec{v}_{\perp} \times \vec{B}_{w}\right) .
\end{aligned}
$$

The input power of the radiation field is determined by the propagating power of the waveguide mode under consideration. At the exit of the wiggler magnet, the energy change of each electron $\Delta \gamma m c^{2}$ is calculated from its interaction with the combined field of the wiggler magnet and the waveguide mode. Specifically to evaluate the energy exchange for individual electrons, we substitute (1) and (3) into (4) to give

$$
\frac{d\left(\gamma m c^{2}\right)}{d t}=-e E_{0} c \beta_{z} \cos \left[\left(k_{z} z-\omega t\right)+\varphi\right]
$$

where $t$ can be determined by

$$
\frac{d t}{d z}=\frac{1}{c \beta_{z}}
$$

and $\beta_{z}$ is evaluated from substituting (1) and (2) into (5) to yield

$$
\begin{aligned}
\frac{d \beta_{z}}{d z}=-\frac{\left(1-\beta_{z}^{2}\right) \omega a_{s}}{\gamma \beta_{z} c} \cos \left[\left(k_{z} z-\omega t\right)\right. & +\varphi] \\
& -\frac{a_{w r}^{2} k_{w}}{\gamma^{2} \beta_{z}} \sin 2 k_{w} z
\end{aligned}
$$

Here, $a_{s}=e E_{0} / m c \omega$ is the dimensionless field strength of the $\mathrm{TM}_{01}$ mode. Choosing the electron spatial location $z$ as the independent variable, we reduce (6) to

$$
\frac{d \gamma}{d z}=-\frac{\omega a_{s}}{c} \cos \left[\left(k_{z} z-\omega t\right)+\varphi\right] .
$$

In our numerical code, (7)-(9) are solved simultaneously using a fourth-order Runge-Kutta-Gill algorithm for electrons with a uniform distribution over one radiation wavelength at the wiggler entrance. For the $\mathrm{TM}_{01}$ mode in cylindrical waveguides, the input power of the radiation field may be determined by its propagating power as follows:

$$
P_{g}=\frac{(\omega / c) k_{z}}{k_{c}^{2}} \frac{E_{0}^{2} A_{e m}}{Z_{0}} J_{1}^{\prime}(2.4048)
$$

where $k_{c}$ is the cutoff wavenumber of the $\mathrm{TM}_{01}$ mode. At the exit of the wiggler magnet, different electrons have different energy changes $\Delta \gamma_{n} m c^{2}$ due to the difference in their initial phases in the radiation field. Hence, the net energy change is the average of energy changes for all electrons $\left\langle\Sigma_{n}\left(\Delta \gamma_{n}\right)\right\rangle m c^{2}$ with $\langle\cdots\rangle$ representing an average over initial electron phases. 
Consequently, the interaction gain can be obtained from the power-gain formula

$$
G=\frac{-\langle\Delta \gamma\rangle m c^{2} I /|e|}{P_{g}}
$$

where $\langle\Delta \gamma\rangle=\left(\Sigma_{n} \Delta \gamma_{n}\right) / n$ with $n$ being the number of electrons in the electron beam. For all numerical examples with monochromatic electron beams in this study, 32 computational charged particles per radiation wavelength were found to be adequate to describe the beam-wave interaction. Hence, the numerical interaction gain is

$$
G_{e}=\frac{-\frac{1}{32} \sum_{n=1}^{32} \Delta \gamma_{n} m c^{2} I /|e|}{P_{g}} .
$$

It is of interest to mention that the run time of our numerical simulation is typically less than $1 \mathrm{~min}$ on a $700-\mathrm{MHz}$ Pentium PC with 128 RAM. Based on this numerical model, the electron interaction with the fields of $\mathrm{TM}_{01}$ mode can be modeled from the small-signal regime to the large-signal regime.

To illustrate saturation behaviors of second-harmonic waveguide FELs, an X-band example $\left(N_{w}=11, E_{b}=\right.$ 66.8-94.7 keV, $I_{b}=20 \mathrm{~mA}$, and $\left.B_{w}=477-1431 \mathrm{G}\right)$ is considered for laser amplification at different values of radiation power within the FEL cavity from the small-signal regime to the large-signal regime. Based on the numerical code developed, the interaction gain is computed and plotted as a function of frequency for four different values of radiation power from $P_{g}=10 \mathrm{~W}$ to $P_{g}=1 \mathrm{~kW}$ in Fig. 2. For comparison, we consider a small wiggler field case $\left(\chi_{0}=0.03\right.$ and $\lambda_{w}=4.18 \mathrm{~cm}$ ) in Fig. 2(a) and a large wiggler field case $\left(\chi_{0}=0.24\right.$ and $\left.\lambda_{w}=3.8 \mathrm{~cm}\right)$ in Fig. 2(b). The choice of these system parameters is partly to ensure the same gain bandwidth (range of percentage frequency interval within which the gain is positive), which has been found to crucially affect gain dependence on key system parameters (e.g., energy-spread). The removal of the influence of gain bandwidth is important to isolate dependence of amplification saturation on various system parameters (e.g., wiggler field, energy spread, etc.). For both cases in Fig. 2, gain bandwidth is $\Delta f / f_{0}=0.19$ ( $f_{0}$ is a central frequency within the range and $8 \mathrm{GHz}$ in this case). This necessitates the need for Fig. 2(a) and (b) to employ different wiggler periods. With gain bandwidth made identical for both cases in Fig. 2, amplification saturation (gain reduction as the radiation power increases) is found to depend on the wiggler field. As the radiation power increases from $10 \mathrm{~W}$ to $1 \mathrm{~kW}$, the interaction in the small wiggler field case $\left[\chi_{0}=0.03\right.$ in Fig. 2(a)] decreases slightly whereas that in the large wiggler case $\left[\chi_{0}=0.24\right.$ in Fig. 2(b)] undergoes a reduction as large as $80 \%$. To put it in a different way, a small wiggler field delays the onset of amplification saturation.

For our comparison of amplification saturation in Fig. 2, we have stressed the importance of maintaining the same gain bandwidth. To confirm the significance of gain bandwidth for amplification saturation, we restudy the small wiggler field case of $\chi_{0}=0.03$ in Fig. 2(a) with a new wiggler period of $3.8 \mathrm{~cm}$ and a reduced gain bandwidth of $\Delta f / f_{0}=0.16$. Again over the same range of the radiation power, interaction gain is plotted in Fig. 3 as a function of frequency. Comparison between Figs. 2(a)

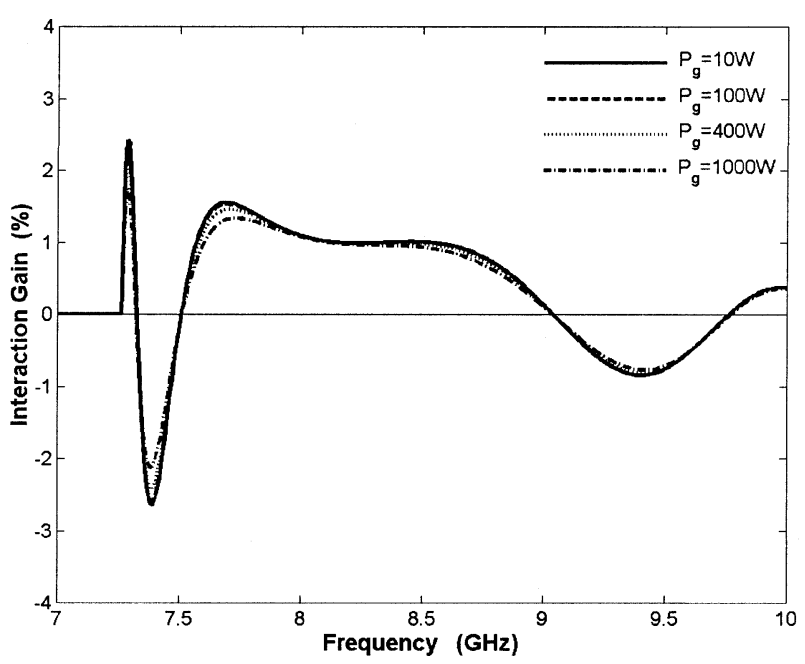

(a)

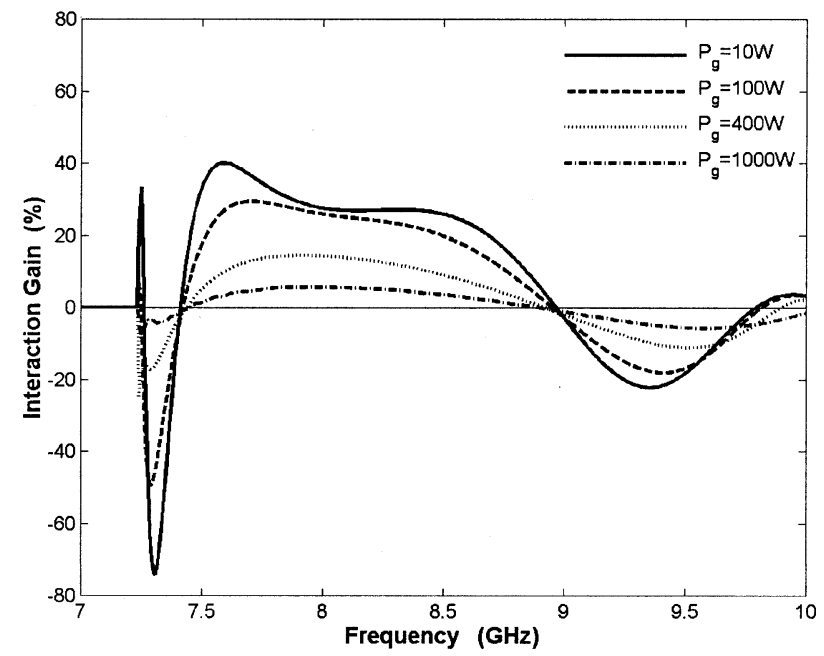

(b)

Fig. 2. Amplification saturation of an X-band second-harmonic waveguide FEL with (a) $\lambda_{w}=4.18 \mathrm{~cm}$ and $\chi_{0}=0.03$; and (b) $\lambda_{w}=3.8 \mathrm{~cm}$ and $\chi_{0}=0.24$.

and 3 suggests severer gain reduction with small bandwidth (in Fig. 3). In other words, small gain bandwidth leads to an earlier onset of amplification saturation. From this standpoint, it is desirable to have as large a gain bandwidth as possible.

To highlight more clearly amplification saturation at different radiation power levels, the interaction gain of the $\mathrm{X}$-band system of Fig. 2 with the same bandwidth $\left(\Delta f / f_{0}=0.19\right)$ is studied at $8 \mathrm{GHz}$ with both small and large wiggler fields. Fig. 4 shows the normalized gain (to the small-signal gain) as a function of in-cavity propagation power. As shown, the interaction gain at large wiggler field starts to saturate earlier at a low in-cavity power, yet its gain degradation speed appears identical to that in the small wiggler field case, thus independent of the wiggler field. The reason why large wiggler field leads to an earlier onset of saturation may be related to the fact that its large small signal gain (at large wiggler field) causes a significant and relatively sudden change in electron energy and as such makes it difficult for the electrons to remain synchronism with their radiation field subsequently as the in-cavity power grows. Also, it is of interest to see effects of gain bandwidth. To this end, interaction gain at $8 \mathrm{GHz}$ of Figs. 2 and 3 is plotted against the in-cavity propagation 


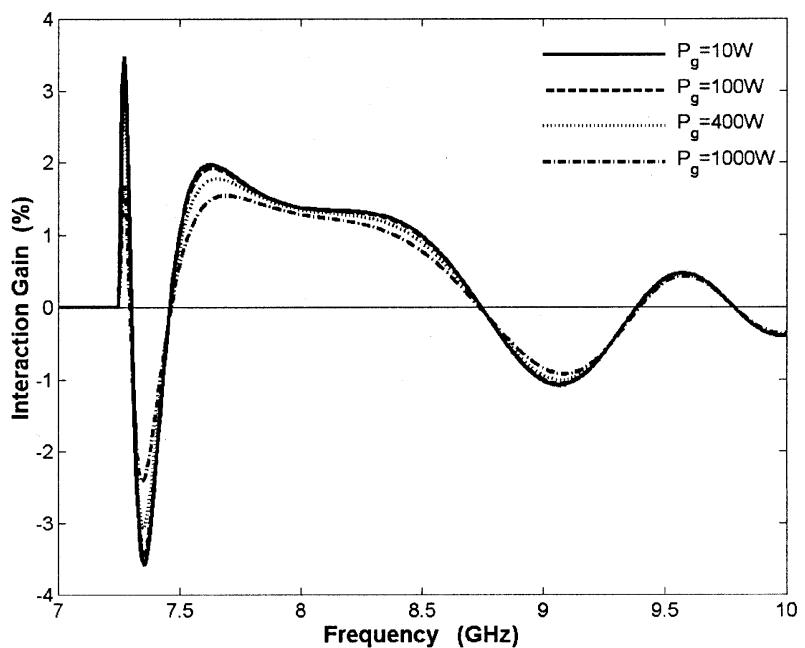

Fig. 3. Amplification saturation of an X-band second-harmonic waveguide FEL with $\lambda_{w}=3.8 \mathrm{~cm}$ and $\chi_{0}=0.03$.

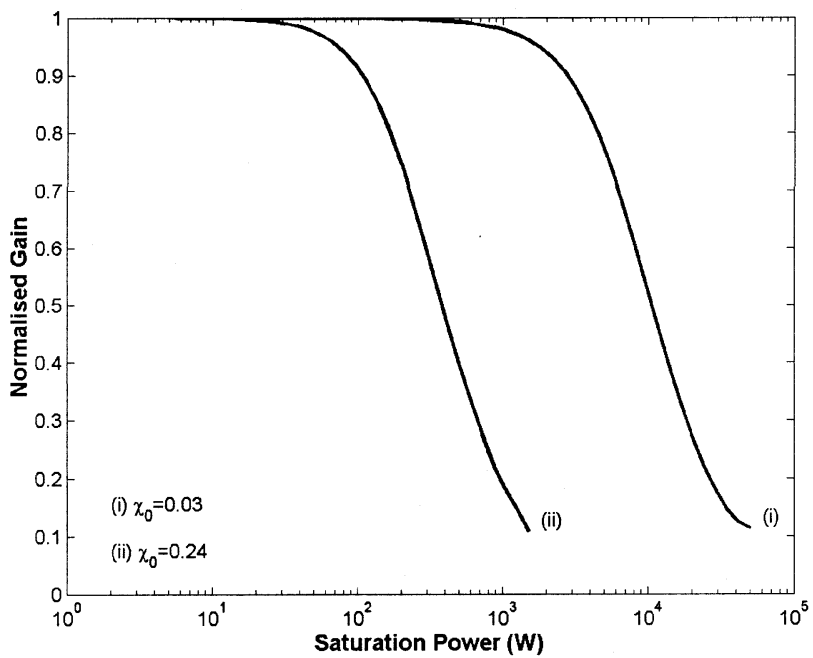

Fig. 4. Comparison between small and large wiggler cases in an X-band second-harmonic waveguide FEL with the same bandwidth of $\Delta f / f_{0}=0.19$.

power in Fig. 5, which illustrates the extent of the effects of gain bandwidth. It is worth mentioning that similar issues are discussed for standard waveguide FELs in literature [4], [5], and [11].

\section{CONDITIONS FOR MAXIMUM POWER OUTPUT}

In general, as the in-cavity power increases, the FEL gain evolves from a large initial value (the small signal gain) and, after a critical onset point, decreases rapidly to a low saturation value at which the laser amplification ceases. At a large wiggler field, this evolution starts at relatively large small-signal gain but decreases much earlier than that with small wiggler field. It is therefore not clear whether a large saturation power (at which laser amplification ceases) is achieved with large or small wiggler field.

A key consideration to evaluate relative merits of small wiggler field and large wiggler field is the roundtrip loss of the radiated electromagnetic wave within the FEL cavity. It should

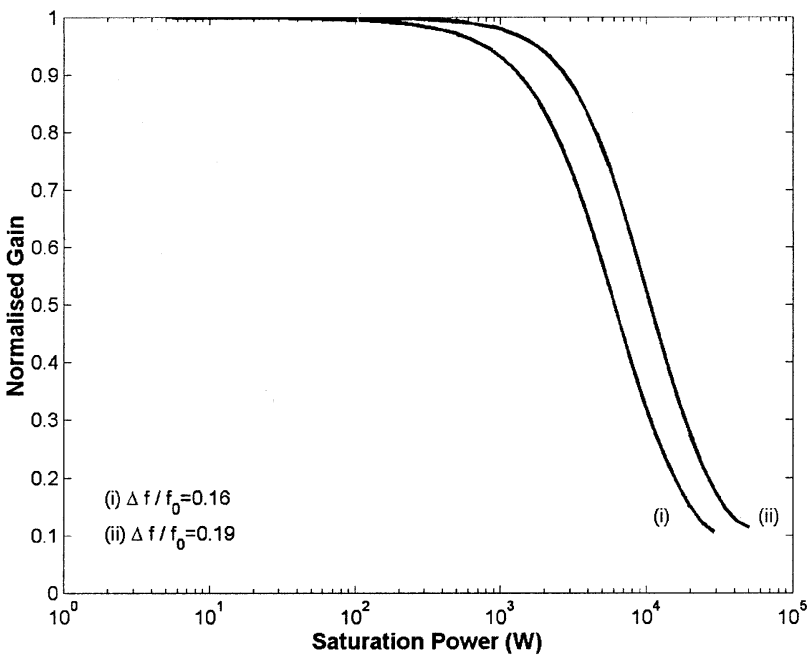

Fig. 5. Gain comparison for X-band FELs with the same wiggler field but different bandwidths of (i) $\Delta f / f_{0}=0.16$ and (ii) $\Delta f / f_{0}=0.19$.

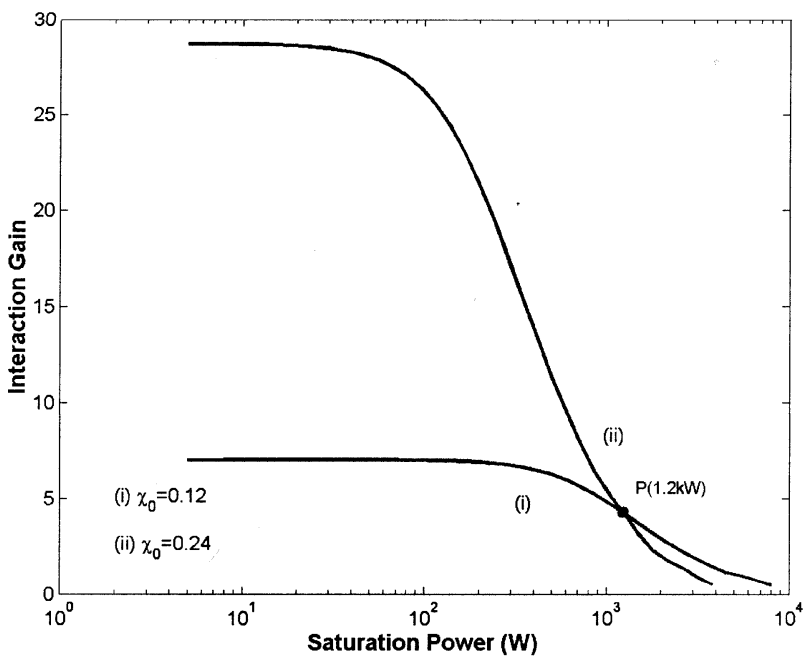

Fig. 6. Gain dependence upon radiation power at small and large wiggler fields in an X-band second-harmonic waveguide FEL of Fig. 3.

be noted that the value of the percentage roundtrip power loss equals to that of the interaction gain at which laser amplification ceases. To illustrate the influence of the roundtrip loss, we note that the two gain-degradation curves in Fig. 6 intersect at an in-cavity power of $1.2 \mathrm{~kW}$ and an interaction gain of $4 \%$. If the roundtrip power loss is greater than $4 \%$, the interaction gain at $\chi_{0}=0.24$ is always greater than that at $\chi_{0}=0.03$ and as such at a roundtrip power loss above $4 \%$, the $\chi_{0}=0.24$ case will saturate at a larger in-cavity power. On the other hand, if the roundtrip power loss is less than $4 \%$, the $\chi_{0}=0.03$ case will saturate at a larger in-cavity power. From these discussions, it is clear that large wiggler field is desirable if the roundtrip power loss is large, whereas very low roundtrip loss favors the option of small wiggler field.

To aid a better assessment of the suitable value of wiggler field, saturation power at four different loss percentages $(0.5 \%$, $1.5 \%, 5 \%$, and $10 \%$ ) is plotted as a function of the wiggler field in Fig. 7. At low power loss (such as $0.5 \%$ and $1.5 \%$ ), the maximum saturation power occurs at small wiggler field 


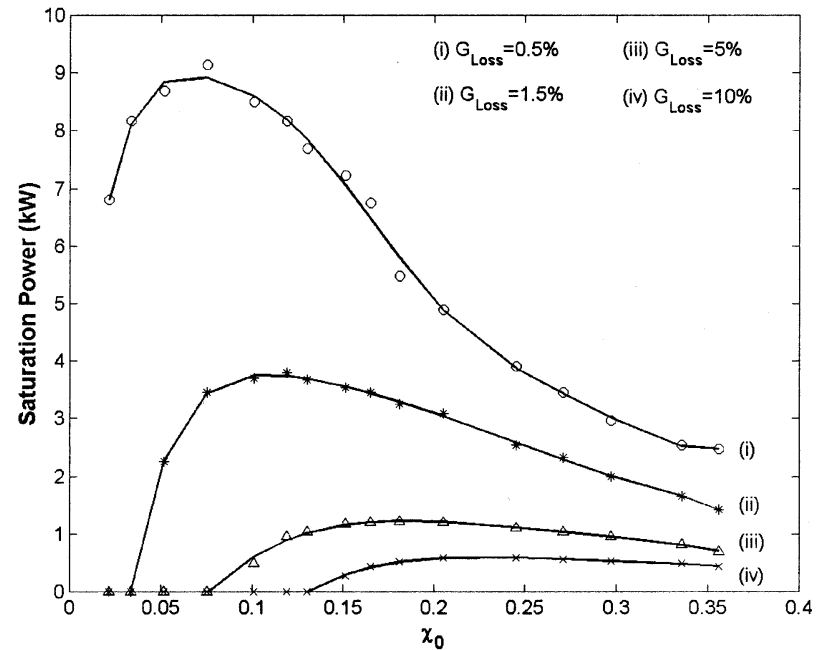

Fig. 7. Saturation power as a function of $\chi_{0}$ at four different loss percentages.

$\left(0.05<\chi_{0}<0.1\right)$ even though their corresponding smallsignal gain is low. As the wiggler field increases $\left(\chi_{0}>0.1\right)$, saturation power reduces, thus confirming the advantage of small wiggler field at small power loss. When the loss is large (above 5\%) on the other hand, low small-signal gain may be inadequate to overcome the roundtrip loss and lasing is not possible (zero saturation power for $\chi_{0}<0.07$ in Fig. 7). As the wiggler field increases, the small-signal gain becomes greater than the power loss and saturation power starts to rise from zero. Their maximum value occurs when $\chi_{0}$ is between 0.14 and 0.19 . This suggests that the maximum saturation power within the FEL cavity occurs at a somewhat middling wiggler field between $\chi_{0}=0.05$ and $\chi_{0}=0.20$, and that large power loss generally requires large wiggler field to maximize the saturation power within the cavity.

In practice, it is also important to know output power to assess its suitability for intended applications and in general it is desirable to attain as large output power as possible at a given electron current. The beam power in all cases may be estimated from multiplying the beam current by the beam voltage and is found in the range of 1.3-1.9 kW. For the beam-wave interaction within an FEL cavity, power output is a component of the roundtrip power loss and a very significant one in most cases (other losses such as wall attenuation can be made very small). From this standpoint, we assume that the roundtrip power loss in the FEL cavity is entirely due to cavity coupling for power output. By multiplying the output coupling to saturation power, we have the output power $P_{\text {output }}=G_{\text {loss }} \times P_{\text {sat }}$ plotted in Fig. 8. For $G_{\text {loss }}=0.5 \%$, the maximum in-cavity saturation power is about $9 \mathrm{~kW}$ and so the output power is $45 \mathrm{~W}$. For $G_{\text {loss }}=1.5 \%$, the maximum in-cavity saturation power reduces to $3.8 \mathrm{~kW}$, but the output power increases to $57 \mathrm{~W}$. Thus, the maximum value of the output power is a tradeoff between saturation power and output coupling. As is shown in Fig. 8, the increment of the maximum point of each curve is in line with the output coupling until when the latter is around 5\%. After that, the maximum output power decreases. The maximum output power occurs in the range of $0.15<\chi_{0}<0.22$ and with $5 \%$ output coupling.

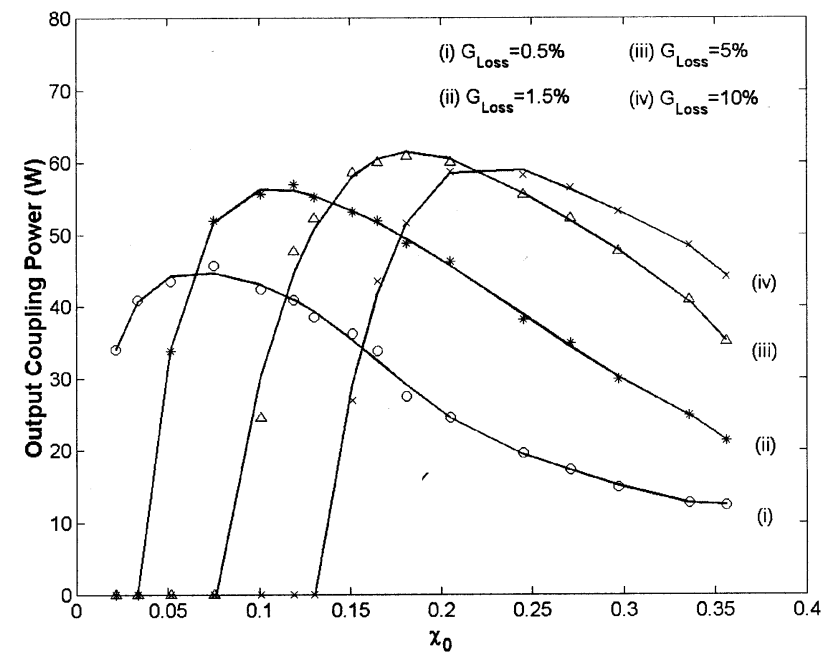

Fig. 8. Output power as a function of $\chi_{0}$ at four different loss percentages.

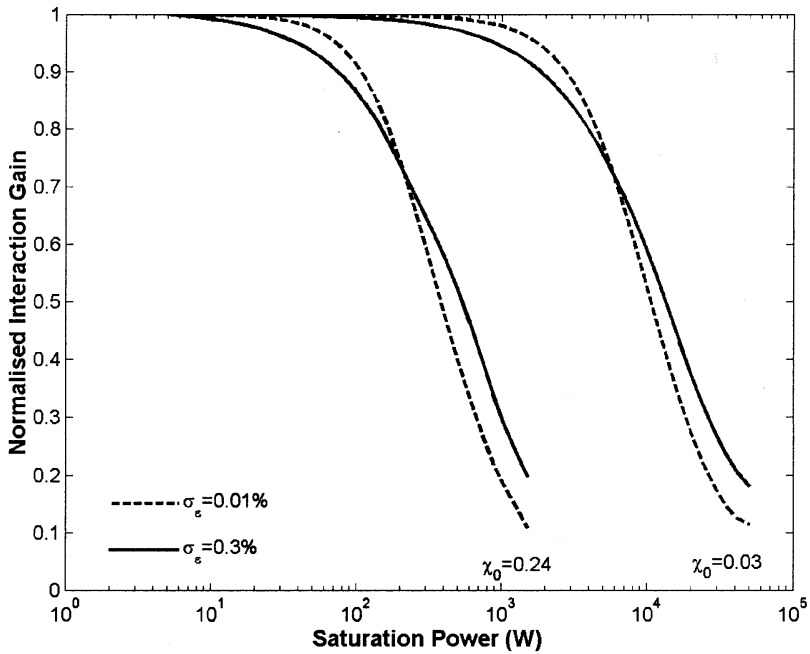

Fig. 9. Normalized interaction gain at $8 \mathrm{GHz}$ with energy spread of $0.01 \%$ (dots) and $0.3 \%$ (solid curve) at small and large wiggler fields.

\section{SATURATion Behaviors With ENERGy Spread}

These analyses are based on the assumption that the electron beam used is monochromatic without any initial energy spread. Here, we will consider the effect of initial electron-energy spread. Mathematically, the electron beam is assumed to have an initial energy spread of a Gaussian distribution as [10]

$$
f(\varepsilon)=\frac{1}{\sqrt{2 \pi} \sigma_{\varepsilon}} \exp \left(-\frac{\varepsilon^{2}}{2 \sigma_{\varepsilon}^{2}}\right)
$$

around its nominal energy, $\gamma_{0} . \sigma_{\varepsilon}$ is the RMS relative energy spread, and

$$
\varepsilon=\frac{\gamma-\gamma_{0}}{\gamma_{0}}
$$

Numerically the energy distribution of (12) is discretized and at each discretized energy at least 32 electrons are required. As an example of the gain degradation due to energy spread, the normalized interaction gain is plotted as a function of the radiation power from $5 \mathrm{~W}$ to $50 \mathrm{~kW}$ in Fig. 9 with two different 


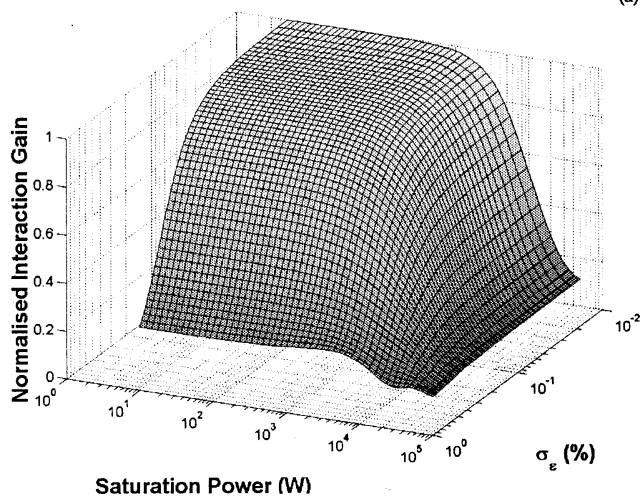

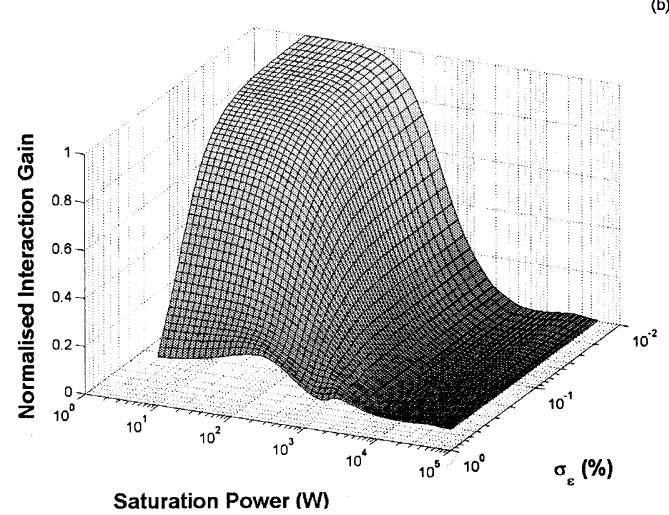

Fig. 10. Radiation power dependence of normalized interaction gain at $8 \mathrm{GHz}$ with energy spread effect in (a) small wiggler field and (b) large wiggler field.

values of energy spread $\left(\sigma_{\varepsilon}=0.01 \%\right.$ and $\left.\sigma_{\varepsilon}=0.3 \%\right)$. It is evident that a large electron-energy spread causes gain degradation to start earlier. For example, in the small wiggler field case $\left(\chi_{0}=0.03\right)$, the onset point (corresponding to $1 \%$ gain reduction from its small-signal value) is changed from $300 \mathrm{~W}$ to $100 \mathrm{~W}$. Similar change (from $30 \mathrm{~W}$ down to $10 \mathrm{~W}$ ) of the saturation onset point is also observed for the large wiggler field $\left(\chi_{0}=0.24\right)$.

In addition to bringing the saturation onset point earlier, an initial electron-energy spread seems to slow down the speed of gain reduction as the radiation increases. For the large wiggler field case $\left(\chi_{0}=0.24\right)$, the gain reduces from $91 \%$ down to $19 \%$ (a difference of $\Delta=72 \%$ ) at $\sigma_{\varepsilon}=0.01 \%$ as radiation power increases from $100 \mathrm{~W}$ to $1 \mathrm{~kW}$. Yet when the energy spread increases to $0.3 \%$, the normalized gain decreases from $86 \%$ down to $30 \%(\Delta=56 \%)$. This lesser gain reduction at large energy spread may result from energy spread causing deviation of electron energy from its nominal value and hence making electrons less sensitive to large in-cavity power. Also observed from Fig. 9 is that the gain-reduction trend seems to be dependent on energy spread and independent of the wiggler field. From this discussion, the energy-spread effects clearly alter both the saturation onset point and the rate of gain reduction due to amplification saturation. These effects are very similar for small and large wiggler fields.

To show the combined effect of radiation power and electron-energy spread on the interaction gain, the interaction gain is normalized and plotted as a function of both the electron-energy spread and the radiation power in Fig. 10. It is shown in Fig. 10 that gain degradation does not appear to be accelerated when both initial electron-energy spread and a significant radiation field $\left(P_{g}>1 \mathrm{~kW}\right)$ are present.

\section{CONCLUSION}

In this paper, second-harmonic waveguide FELs were studied in the large signal regime for both small and large wiggler fields. It was found that amplification saturation is less severe with small wiggler field than that with large wiggler field and that large gain bandwidth can further delay the onset of amplifica- tion saturation. For an FEL cavity with low roundtrip power loss, the maximum saturation power was found in the range of $0.05<\chi_{0}<0.1$. For greater losses, the maximum saturation power moves to the $0.18<\chi_{0}<0.2$ range. Beyond $\chi_{0}>0.2$, the saturation power decreases gradually. By considering the output coupling as the roundtrip power loss, the maximum attainable output power was also obtained. Furthermore, energy-spread effects have been found to alter both the saturation onset point and the gain-reduction rate but remain relatively independent of wiggler field.

\section{REFERENCES}

[1] J. F. Schmerge, W. J. W. Lewellen, Y. C. Huang, J. Feinstein, and R. H. Pantell, "The free-electron laser as a laboratory instrument," IEEE J. Quantum Electron., vol. 31, pp. 1166-1171, June 1995.

[2] G. Kong, J. Lucas, and R. A. Stuart, "Low voltage ubitrons for industrial purposes," Nucl. Instrum. Methods Phy. Res. A, Accel. Spectrom. Detect. Assoc. Equip., vol. 304, pp. 238-242, 1991.

[3] Y. Y. Kawamura, B. C. Lee, M. Kawai, and K. Toyoda, "Various characteristics of a waveguide mode free-electron laser using a long-pulse relativistic electron-beam," Phys. Rev. E, Stat. Phys. Plasmas Fluids Relat. Interdiscp. Top., vol. 47, pp. 4399-4404, 1993.

[4] F. Ciocci, R. Bartolini, A. Doria, G. P. Gallerano, E. Giovenale, M. F. Kimmitt, G. Messina, and A. Renieri, "Operation of a compact freeelectron laser in the millimeter-wave region with a bunched electronbeam," Phys. Rev. Lett., vol. 70, pp. 928-931, 1993.

[5] D. E. Pershing, R. D. Seeley, R. H. Jackson, and H. P. Freund, "Amplifier performance of the NRL ubitron," Nucl. Instrum. Methods Phy. Res. A, Accel. Spectrom. Detect. Assoc. Equip., vol. 358, pp. 104-107, 1995.

[6] J. H. Brownell, J. Walsh, and G. Doucas, "Spontaneous Smith-Purcell radiation described through induced surface currents," Phys. Rev. E, Stat. Phys. Plasmas Fluids Relat. Interdiscp. Top., vol. 57, pp. 1075-1080, 1998.

[7] M. G. Kong, "A low voltage waveguide free electron laser based on a longitudinal interaction mechanism," Opt. Commun., vol. 141, pp. 48-52, 1997.

[8] X. Zhong and M. G. Kong, "Effects on nonresonant electron velocity components in a second harmonic waveguide free electron laser," Opt. Commun., vol. 168, pp. 375-381, 1999.

[9] —-, "Strong wiggler field assisted amplification in a second harmonic waveguide free electron laser," IEEE Trans. Plasma Sci., vol. 30, pp. 630-638, Apr. 2002.

[10] M. G. Kong, "Energy spread analysis of a waveguide optical klystron," Opt. Commun., vol. 132, pp. 464-468, 1996.

[11] H. P. Fruend and T. M. Antonsen Jr., Principles of Free-Electron Lasers. London, U.K.: Chapman \& Hall, 1992. 


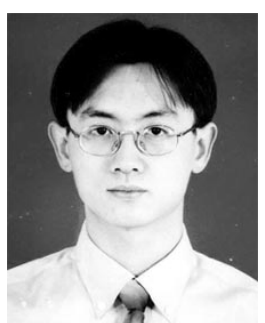

Xiehe Zhong ( $\mathrm{S}^{\prime}$ 01) was born in Shenzhen, China, in 1975. He received the B.Sc. degree in electronic engineering from Shenzhen University, China, in 1997, the M.Sc. degree in telecommunications engineering from Liverpool University, Liverpool, U.K., in 1998, and the $\mathrm{Ph} . \mathrm{D}$. degree in electrical engineering from Loughborough University, U.K., in 2002.

He is currently a Research Assistant at Loughborough University. His research interests include compact waveguide free electron lasers and optical communications systems.

Dr. Zhong is an Associate Member of the IEE (U.K.).

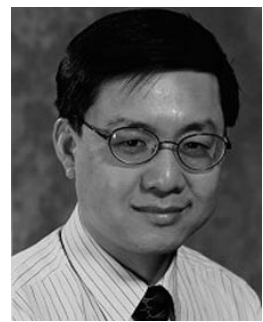

Michael G. Kong (M'94-SM'98) received the B.Sc. and M.Sc. degrees, in electronics engineering, from Zhejiang University, China, in 1984 and 1987, respectively, and the Ph.D. degree in electrical engineering from Liverpool University, Liverpool, U.K., in 1992.

He has been actively involved in research on compact waveguide free electron lasers, nonthermal atmospheric gas discharges, and power systems and devices. In these subject areas, he has published some 100 papers in scientific journals and peer-reviewed conference proceedings. He has been a Senior Lecturer at Loughborough University, U.K., since 1999 and, prior to that, he was a Lecturer at Liverpool University from 1995 to 1999. He is a Chief Examiner for the Engineering Council, UK.

Dr. Kong is a Committee Member of the Ion and Plasma Surface Interaction Group at the Institute of Physics, U.K. 